Agronomía Costarricense 39(3): 21-36. ISSN:0377-9424 / 2015

www.mag.go.cr/rev_agr/index.html www.cia.ucr.ac.cr

\title{
INOCULACIÓN AL SUELO CON Pseudomonas fluorescens, Azospirillum oryzae, Bacillus subtilis Y MICROORGANISMOS DE MONTAÑA (MM) Y SU EFECTO SOBRE UN SISTEMA DE ROTACIÓN SOYA-TOMATE BAJO CONDICIONES DE INVERNADERO
}

\author{
Leida Castro Barquero ${ }^{1 / *}$, Mariana Murillo Roos ${ }^{*}$, Lidieth Uribe Lorío*, Rafael Mata Chinchilla*
}

Palabras clave: Azospirillum oryzae; Pseudomonas fluorescens; Bacillus subtilis; microorganismos de montaña (MM); inoculación.

Keywords: Azospirillum oryzae; Pseudomonas fluorescens; Bacillus subtilis; microorganismos de montaña (MM); inoculation.

Recibido: 10/04/15

\section{RESUMEN}

Se evaluó un sistema de rotación soyatomate, con incorporación de biomasa verde y aplicación de inóculos microbianos individuales y en mezcla sobre el crecimiento de las plantas y propiedades edáficas; para ello se evaluaron en invernadero por 24 meses los siguientes 9 tratamientos: solo tomate $(\mathrm{T})$; rotación tomate-soya (TS); rotación tomate-soya con inoculaciones individuales de Azospirillum oryzae (A); de Pseudomonas fluorescens (P); de Bacillus subtilis (B); de microorganismos de montaña (MM); y las inoculaciones en mezcla de $B$. subtilis y $P$. fluorescens (BP); de B. subtilis, P. fluorescens y A. oryzae (BPA); de B. subtilis, P. fluorescens, Azospirillum sp. y MM (BPAMM). Se evaluaron las variables físicas: densidad aparente y de partículas; conductividad hidráulica; poros totales; estabilidad de agregados; resistencia a la penetración (RP); las variables químicas: $\mathrm{pH}$; conductividad eléctrica; contenido de $\mathrm{N}$ y $\mathrm{C}$; relación $\mathrm{C} / \mathrm{N}$; contenido de nutrientes en suelos y foliares; las variables biológicas: respiración de suelos, unidades formadoras de colonias de hongos, bacterias y actinomicetos y el peso fresco y seco foliar. Las variables físicas no fueron

1 Autor para correspondencia. Correo electrónico: leida.castro@ucr.ac.cr
Aceptado: 01/06/15

\begin{abstract}
Soil inoculation with Pseudomonas fluorescens, Azospirillum oryzae, Bacillus subtilis and mountain microorganisms (MM) and its effect on a soybean-tomato crop rotation system under greenhouse conditions. The effect, on growth of plants and soil properties, of incorporating green biomass and applying individual and compound microbial inocula was evaluated in a soybeantomato crop rotation system during 24 months in the greenhouse. Nine different conditions were evaluated: tomato alone $(\mathrm{T})$; tomato-soybean rotation (TS); tomato-soybean rotation with individual inoculations of Azospirillum oryzae (A); of Pseudomonas fluorescens (P); of Bacillus subtilis (B); of mountain microorganisms (MM); and tomato-soybean rotation with mixtures of $B$. subtilis and P. fluorescens (BP); of B. subtilis, $P$. fluorescens, and A. oryzae (BPA); of B. subtilis, $P$. fluorescens, Azospirillum sp., and mountain microorganisms (BPAMM). The following physical and chemical soil indicators (variables) were evaluated: apparent density; particle density; hydraulic conductivity; pore volume (porosity); aggregate stability; penetration resistance (PR);
\end{abstract}

Centro de Investigaciones Agronómicas, Universidad de Costa Rica. 
afectadas por los tratamientos, con excepción de $\mathrm{RP}$, que fue mayor en el tratamiento T. Las variables biológicas y químicas fueron sensibles a los tratamientos, con valores significativamente más altos en presencia de MM. Destaca el incremento del $\mathrm{P}$ en solución de suelo en tratamientos a los que se adicionó MM: pasó de 6 a $20 \mathrm{mg} . \mathrm{l}^{-1}$; esta condición se reflejó además en la cantidad de $\mathrm{P}$ en el tejido foliar al final del ensayo. Se determinó que el pH, CE y la respiración del suelo fueron afectados por la interacción entre los tratamientos aplicados y el tiempo transcurrido; los mayores valores se obtuvieron al final del ensayo y en los tratamientos con MM.

\section{INTRODUCCIÓN}

El suelo es el ecosistema más diverso y complejo del planeta y proporciona un amplio rango de los recursos que las plantas necesitan para crecer y enfrentar diversos retos bióticos y abióticos. Debido a las relaciones existentes entre plantas, suelos, microorganismos y ambiente, la complejidad dinámica del suelo involucra factores físicos, químicos y biológicos, en una red de interacciones que facultan la transferencia de energía, los ciclos de materiales y nutrientes y la transmisión de información. Todos estos factores interactúan a diferentes niveles de organización y siempre de manera compleja, debido a ello, las prácticas de manejo agrícola, las cuales distorsionan el equilibrio natural de los ecosistemas, afectan la estructura y función del suelo a corto, mediano y largo plazo (Bautista et ál. 2008, Young y Crawford 2004, Jones y Hinsinger 2008, Mitter et ál. 2013).

Las actividades antropogénicas de uso convencional, principalmente a partir de la
$\mathrm{pH}$; electrical conductivity (EC); carbon and nitrogen content; $\mathrm{C} / \mathrm{N}$ ratio; and soil and foliar nutrient content. Biological indicators were also assessed: soil respiration; colony-forming units (CFU) of fungi, bacteria and actinomycetes; fresh and dry foliar weight. The physical variables were not affected by the treatments, except for PR, which was higher in treatment T. Chemical and biological variables were sensitive to the treatments, with significantly higher values in the presence of MM. The rise of $\mathrm{P}$ in soil solution in treatments with MM was of special importance: it increased from 6 to $20 \mathrm{mg} .1^{-1}$; this condition was also reflected in the concentration of $\mathrm{P}$ in foliar tissue at the end of assay. It was determined that $\mathrm{pH}, \mathrm{EC}$ and soil respiration were affected by the interaction among the treatments applied and the time elapsed; the highest values were obtained at the end of the assay and in treatments with MM.

revolución verde, como el monocultivo, la labranza intensiva y el uso de agroquímicos pueden afectar negativamente la calidad y productividad de los suelos, por lo que un mejor entendimiento de las relaciones que se dan en los agrosistemas, podría permitir el desarrollo de estrategias para restaurar suelos agotados, que debido a la pérdida de sus propiedades holísticas y sistémicas han reducido su heterogeneidad y en consecuencia su estabilidad ecológica (Capra 1998, Chaparro et ál. 2012, Kibblewhite et ál. 2015).

Hoy en día el desarrollo de una agricultura sostenible es vital no solo para hacer frente a la demanda de alimentos y fibras sino para mantener otros servicios ambientales necesarios para la vida (Godfray et ál. 2010, Kibblewhite et ál. 2015). El uso de prácticas de conservación como la labranza mínima, la rotación de cultivos, la incorporación de residuos y el uso de leguminosas, representan una oportunidad de capturar $\mathrm{C}$ y $\mathrm{N}$ atmosférico en el suelo, que permite su conservación física y biológica (Balota et ál. 
2004, Govaerts et ál. 2007) mientras se mitiga el incremento en la concentración de $\mathrm{CO}_{2}$ en la atmósfera y los cambios negativos a escala global en los ciclos de nutrientes y agua (Ehrenfeld et ál. 2005).

Las comunidades microbianas en los suelos se consideran vitales para asegurar la sostenibilidad de los ecosistemas (Young y Crawford 2004, Barrios 2007, Van der Heijden et ál. 2008, Eisenhauer et ál. 2010, Mendes et ál. 2011, Chaparro et ál. 2012), donde son responsables de funciones tales como: las transformaciones de carbono, el ciclaje de nutrientes, el mantenimiento de la estructura del suelo y la regulación de las poblaciones biológicas (Castro et ál. 2009, Singh et ál. 2011, Kibblewhite et ál. 2015). En los agrosistemas estas funciones tienen una influencia permanente sobre la salud y la productividad de los cultivos. Se han encontrado como parte integral de la biota rizosférica de cultivos agrícolas un amplio rango de bacterias de vida libre, simbióticas o en asociación de géneros tales como: Acinetobacter, Alcaligenes, Arthrobacter, Azospirillum, Azotobacter, Bacillus, Burkholderia, Enterobacter, Erwinia, Flavobacterium, Proteus, Pseudomonas, Rhizobium, Serratia, y Xanthomonas entre otras (Bhattacharyya y Jha 2012).

En los últimos años se ha destacado el éxito de la inoculación con microorganismos para la promoción del crecimiento vegetal y el control biológico de patógenos (Vessey 2003). Los géneros bacterianos Azospirillum, Bacillus y Pseudomonas han sido ampliamente utilizados con estos objetivos (Bhattacharyya y Jha 2012).

Debido a la gran variedad de funciones realizadas por microorganismos diferentes, las inoculaciones múltiples pueden ser más efectivas que las inoculaciones individuales. Con la adición de inoculaciones combinadas de bacterias promotoras de crecimiento se ha logrado aumentar la absorción de nutrientes y el crecimiento de las plantas, además de conferir resistencia a estrés abiótico y suprimir enfermedades y plagas (Kirankumar et ál. 2008, Cummings 2009, Guiñazú et ál. 2009, Dimkpa et ál. 2009, De Vleesschauwer y Höfte 2009).
Torsvik y Øvreås (2002) y Kibblewhite et ál. (2015) destacan que la diversidad microbiana y los procesos en el suelo son llevados a cabo por "consorcios microbianos" cuya característica principal es la diversidad funcional más que la de grupos taxonómicos, por lo que las funciones individuales en estos consorcios quedan traslapadas, este hecho es de gran importancia para la estabilidad y resiliencia del ecosistema suelo.

La elaboración de Microorganismos de Montaña (MM) se fundamenta en la tecnología desarrollada en los años 80 por el japonés Dr. Teruo Higa, conocida como Microorganismos Eficientes (EM) (Mayera et ál. 2010), Los MM se podrían catalogar como "consorcios microbianos" ya que su composición y las posibles relaciones que generan son múltiples, se indica que contienen bacterias fotosintéticas, bacterias productoras de ácido láctico, actinomicetes, hongos filamentosos y levaduras. Los MM son un producto de fabricación artesanal de bajo costo, que no requiere medios de crecimiento sofisticados para el escalamiento y que pretende aprovechar la diversidad microbiana tanto taxonómica como funcional, de las comunidades de microorganismos nativos de zonas boscosas, para luego incorporarlos en las unidades de producción agrícola. Entre los usuarios de este tipo de tecnología se acepta que la mejor fuente de inoculo son los bosques cercanos a los sitios de producción agrícola, ya que presentan microorganismos adaptados a las condiciones de la zona. Existe poca literatura que respalde las respuestas positivas del uso de este tipo de inoculo en la producción, se destaca el aporte de Restrepo y Hensel 2007.

La presente investigación tuvo como objetivo evaluar en un sistema de rotación tomatesoya con incorporación de biomasa verde, la aplicación de inóculos individuales y en mezcla de Azospirillum, Pseudomonas, Bacillus y bioles de Microorganismos de Montaña, sobre el crecimiento de las plantas y variables edáficas.

\section{MATERIALES Y MÉTODOS}

El experimento se realizó en el invernadero y Laboratorio de Microbiología Agrícola 
del Centro de Investigaciones Agronómicas de la Universidad de Costa Rica entre julio del 2011 y octubre del 2013. Se evaluaron los siguientes 9 tratamientos, a partir de 4 repeticiones: 1-Solo tomate (T); 2-Rotación tomate-soya (TS); 3-TS con inoculación de Azospirillum oryzae (A); 4-TS con inoculación de Pseudomonas fluorescens (P); 5-TS con inoculación de Bacillus subtilis (B); 6-TS con inoculación de Microorganismos de Montaña (MM); 7-TS con inoculación de B. subtilis y $P$. fluorescens (BP); 8- TS con inoculación de B. subtilis, P. fluorescens y A. oryzae (BPA); 9-TS con inoculación de B. subtilis, P.fluorescens, A. oryzae y MM (BPAMM). Las cepas microbianas utilizadas pertenecen a la colección del Laboratorio de Microbiología Agrícola del Centro de Investigaciones Agronómicas de la Universidad de Costa Rica.

Se utilizó suelo proveniente de Liberia Guanacaste clasificado como Lithic Ustorthents (Entisol pedregoso de régimen ústico, derivado de ignimbritas ácidas) con las características que se muestran en el Cuadro 1. El suelo se transportó al invernadero del Centro de Investigaciones Agronómicas donde se procedió a cribar con un sarán grueso para eliminar rocas u otros objetos, posteriormente se llenaron todos los recipientes pertenecientes al ensayo con $8 \mathrm{~kg}$ de suelo (cada recipiente correspondió a una unidad experimental) y se cultivaron con soya (var. CIGRAS 06), excepto el tratamiento de solo tomate, cuyos recipientes se dejaron sin cultivar. En el siguiente ciclo de cultivo (o rotación) se sembró tomate (var. Hayslip) en todos los tratamientos, en el tercer y cuarto ciclo de cultivo se sembró soya (excepto en el tratamiento de solo tomate), finalmente en el quinto ciclo se plantó tomate en todos los tratamientos. Posterior a la siembra del primer ciclo, el suelo no fue disturbado, excepto para la toma de muestras, las arvenses que crecieron espontáneamente se cortaron con tijera y se dejaron sobre la superficie de los recipientes (Figura 1). El suelo en las macetas se mantuvo siempre húmedo y se

Cuadro 1. Análisis químico del suelo utilizado en el experimento.

\begin{tabular}{|c|c|c|c|c|c|c|c|c|c|c|c|c|c|c|}
\hline \multirow{3}{*}{$\begin{array}{c}\mathrm{pH} \\
\mathrm{H}_{2} \mathrm{O}\end{array}$} & \multicolumn{14}{|c|}{ Análisis } \\
\hline & \multicolumn{5}{|c|}{$\operatorname{Cmol}(+) \cdot 1^{-1}$} & \multirow{2}{*}{$\begin{array}{c}\% \\
\text { SA }\end{array}$} & \multicolumn{5}{|c|}{$\mathrm{mg} . \mathrm{l}^{-1}$} & \multicolumn{2}{|c|}{$\%$} & \multirow[b]{2}{*}{$\mathrm{C} / \mathrm{N}$} \\
\hline & acidez & $\mathrm{Ca}$ & $\mathrm{Mg}$ & K & CICE & & $\mathrm{P}$ & $\mathrm{Zn}$ & $\mathrm{Cu}$ & $\mathrm{Fe}$ & $\mathrm{Mn}$ & $\mathrm{C}$ & $\mathrm{N}$ & \\
\hline 5,5 & 0,14 & 7,22 & 1,38 & 0,51 & 9,25 & 2,0 & 6,0 & 8,0 & 3,0 & 275 & 115 & 1,83 & 0,17 & 10,8 \\
\hline
\end{tabular}
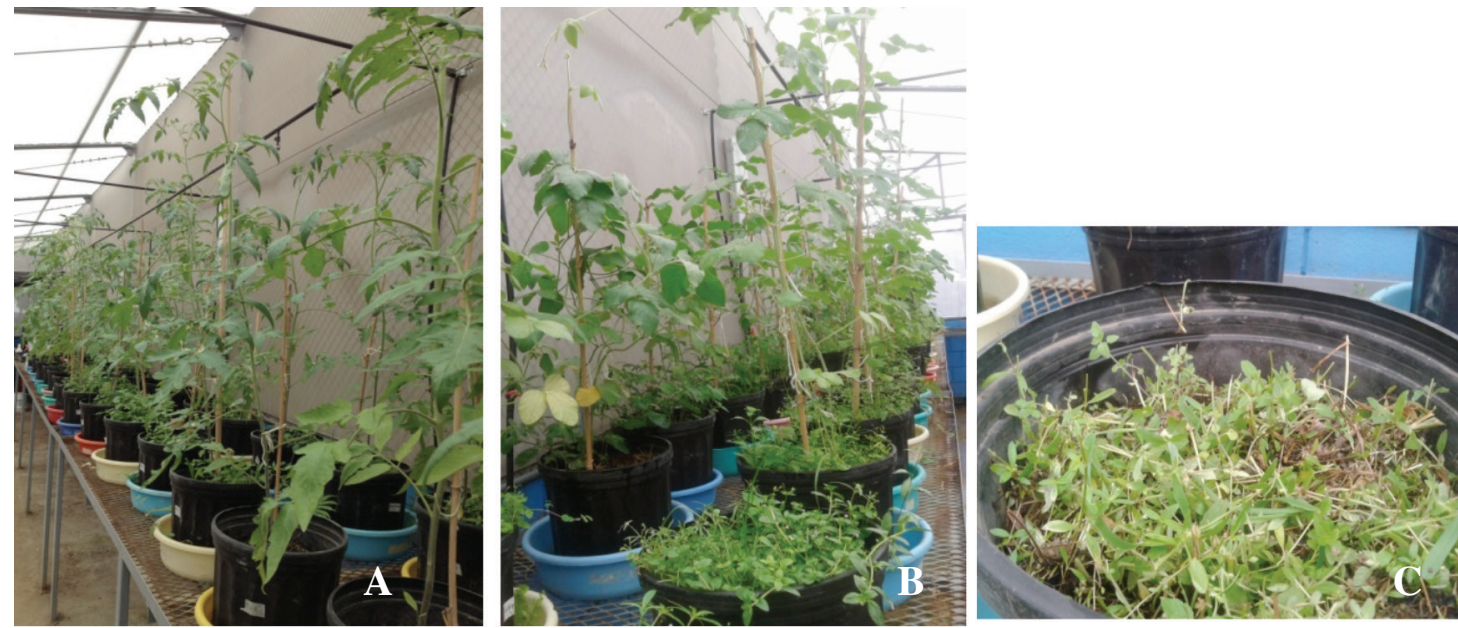

Fig.1. Rotación soya-tomate y manejo de arvenses. A-Tomate, B- soya, C-Manejo de arvenses. 
observó un crecimiento adecuado de las plantas durante todas las rotaciones realizadas. No se hicieron aplicaciones de ningún tipo de fertilización química.

En los tratamientos con soya, se cosechó el material foliar a los 3 meses después de la siembra, las muestras de cada tratamiento se pesaron individualmente, se mezclaron y se aplicaron como abono verde, a partir de la distribución del material verde por partes iguales en cada una de las repeticiones de cada tratamiento, este material se dejó sobre la superficie de las macetas y aproximadamente 2 meses después se trasplantaron plántulas de tomate de 30 días de edad. Dos meses después las plantas se cosecharon y se les determinó el peso fresco y seco foliar, el material seco fue incorporado a las macetas de la misma forma que la soya, con la humedad adecuada para su descomposición por aproximadamente 2 meses (Figura 1). Se realizaron en cada ciclo de cultivo, 2 aplicaciones de los inóculos microbianos según correspondiese; para ello se colocaron en cada unidad experimental a nivel de suelo y alrededor del tallo, $10 \mathrm{ml}$ de una suspensión en agua estéril de cada una de las bacterias, cultivadas por 48 horas en agar nutritivo. Se utilizó una concentración bacteriana de 5*108 UFC.ml-1 para A. oryzae, $1,2 * 10^{9}$ UFC.ml ${ }^{-1}$ para $P$. fluorescens y $2 * 10^{7}$ UFC.ml ${ }^{-1}$ para $B$. subtillis.

A la siembra se inocularon las semillas de soya con $2 \mathrm{ml}$ de una suspensión en agua estéril de una mezcla de 3 cepas de Bradyrhizobium japonicum (CR-506, CR-508 CR-514), pertenecientes a la colección del Laboratorio de Microbiología Agrícola, la concentración de las bacterias inoculadas fue de $8 \times 10^{8}$ UFC. $\mathrm{ml}^{-1}$.

Los MM sólidos para la preparación de los bioles se elaboraron de la siguiente manera: En un estañón con tapa hermética, válvula de salida de gases y con capacidad de 200 litros, se colocó la mezcla de 2 sacos de mantillo de bosque con un saco de semolina de arroz y 2 litros de melaza, el material se comprimió en el recipiente y se tapó por un mes. Posteriormente esta mezcla sólida se utilizó para preparar un biol líquido, para ello se colocaron en un recipiente de plástico oscuro y tapa de rosca, 8 litros de agua de manantial, 300 ml de melaza y $400 \mathrm{~g}$ de MM sólido, se mezcló y se dejo en reposo para su posterior aplicación. Las aplicaciones de MM líquido se realizaron 12 y 26 días después de la preparación. Se aplicaron 250 $\mathrm{ml}$ de MM líquido por maceta, diluido al $50 \%$ con agua de riego, para un volumen final de $500 \mathrm{ml}$.

Se determinó la densidad aparente de partículas, la conductividad hidráulica, la porosidad total y la resistencia a la penetración, mediante las metodologías propuestas por Forsythe (1975) y Henríquez y Cabalceta (2012) y la estabilidad de los agregados a partir del método descrito por Kemper (1965). Estas determinaciones se realizaron en el Laboratorio de Recursos Naturales del Centro de Investigaciones Agronómicas (CIA). La biomasa microbiana se evaluó mediante la metodología de extracción-fumigación de Vance et ál. (1987), para la determinación de la respiración de suelo se utilizó la técnica de Jenkinson y Powlson (1976), las unidades formadoras de colonias (UFC) de hongos, bacterias y actinomicetes se determinaron mediante la técnica de recuentos de microorganismos viables en placas petri, el peso fresco y seco foliar y peso fresco radical se realizaron en el Laboratorio de Microbiología Agrícola del CIA. La determinación de $\mathrm{N}$ y C del suelo y la relación C/N se llevó a cabo por el método de Bremner y Tabatabai (1971), el contenido foliar de nutrientes por los métodos descritos por Díaz y Hunter 1978 y Henríquez y Cabalceta (2012), pH y conductividad eléctrica con un potenciómetro-conductímetro portátil, Metter Toledo, Seven go duo en el Laboratorio de Suelos y Foliares del CIA.

Los tratamientos evaluados se arreglaron en un diseño irrestricto al azar. Los datos se trataron por medio de análisis de varianza y se utilizó como separador de medias la prueba de LSD Fisher al 5\%. Las variables que presentaron interacción del tiempo con los tratamientos, se analizaron por medio de un diseño factorial con los factores tratamientos y tiempo (T1: evaluación al final del primer ciclo de soya; T2: evaluación al final del último ciclo de soya). 


\section{RESULTADOS Y DISCUSIÓN}

\section{Evaluación del sistema de rotación soya- tomate}

En cuanto a las características físicas del suelo, la resistencia a la penetración (RP) fue la única variable afectada por los tratamientos (Cuadro 2); en este caso, solamente el tratamiento $\mathrm{T}$ en $\mathrm{T} 2$ que es la evaluación al final del último ciclo de soya, obtuvo un valor estadísticamente mayor $(\mathrm{p}=0,0031)$ lo que indica que la incorporación de la biomasa de la soya afectó en todos los casos, el valor de esta variable, aspecto que coincide con lo indicado por Martínez (2008) que suelos con mayor contenido de materia orgánica tienen a presentar una menor RP.

Cuadro 2. Resistencia a la penetración del suelo mostrada al final del primer ciclo de soya (T1) y al final del último ciclo de soya (T2).

\begin{tabular}{c|cccccccccc}
\hline \multirow{2}{*}{$\begin{array}{c}\text { Resistencia a la } \\
\text { penetración } \\
\left(\mathrm{kg} \mathrm{cm}^{2}\right)\end{array}$} & $\mathrm{T}$ & $\mathrm{TS}$ & $\mathrm{B}$ & $\mathrm{P}$ & $\mathrm{A}$ & $\mathrm{MM}$ & $\mathrm{BP}$ & BPA & BPAMM \\
\cline { 3 - 8 } $\mathrm{T} 1$ & $0,80 \mathrm{a}$ & $0,38 \mathrm{a}$ & $0,43 \mathrm{a}$ & $0,45 \mathrm{a}$ & $0,59 \mathrm{a}$ & $0,46 \mathrm{a}$ & $0,45 \mathrm{a}$ & $0,31 \mathrm{a}$ & $0,43 \mathrm{a}$ \\
$\mathrm{y}$ & $1,01 \mathrm{~b}$ & $0,39 \mathrm{a}$ & $0,46 \mathrm{a}$ & $0,44 \mathrm{a}$ & $0,49 \mathrm{a}$ & $0,45 \mathrm{a}$ & $0,48 \mathrm{a}$ & $0,50 \mathrm{a}$ & $0,46 \mathrm{a}$ \\
\hline
\end{tabular}

Diferentes letras implican diferencias significativas entre los tratamientos. $(\mathrm{p}=0,0031)$.

Los contenidos de $\mathrm{C}$ y $\mathrm{N}$ en el suelo (Figuras 2 y 3 ) aumentaron con el paso del tiempo en todos los tratamientos $(\mathrm{p}=<0,0001)$. El mayor incremento comparado al contenido inicial (1,83\% para C y $0,17 \%$ para N) se presentó en el tratamiento de mayor complejidad microbiana (BPAMM), y fue de alrededor del $100 \%$ para ambos elementos. Cambios en los contenidos de $\mathrm{C}$ y $\mathrm{N}$ a corto plazo han sido observados por diferentes autores (Sauerbeck y González 1977,
Montagnini et ál. 1993, Wood and Lawrence 2008), sin embargo Hartemink (2003) considera que los mismos deben mantenerse al menos por 10 años para ser considerados como estables. Marín (2015) encontró que suelos sometidos a un manejo orgánico por 15 años presentaron un contenido de 6,24\% de $\mathrm{C}$ y $0,59 \%$ de $\mathrm{N}$, mientras que suelos aledaños utilizados en agricultura convencional presentaron valores de $4,05 \%$ de C y $0,41 \%$ de $\mathrm{N}$. 


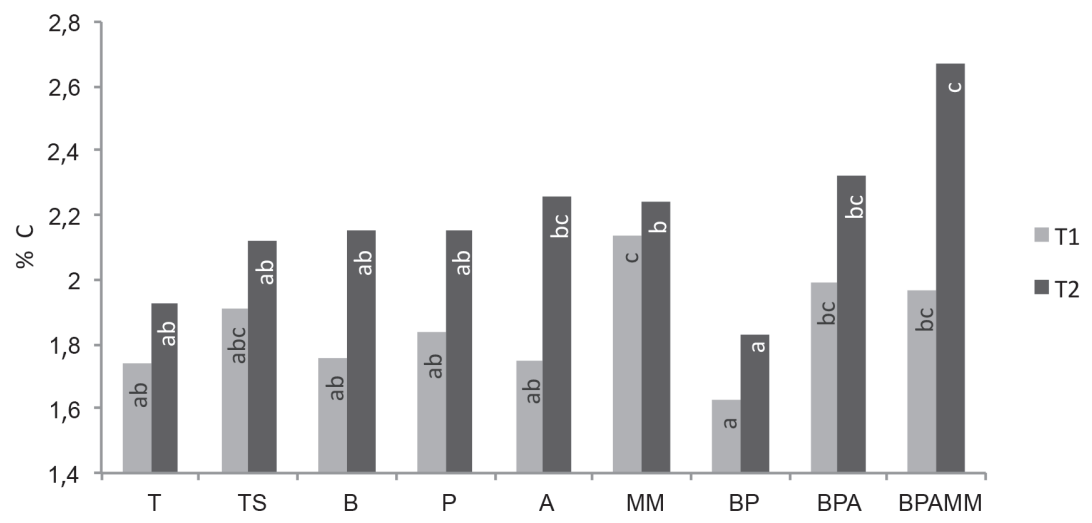

Barras del mismo color con diferentes letras implican diferencias significativas entre los tratamientos.

Fig. 2. Porcentaje de $\mathrm{C}$ del suelo al final del primer ciclo de soya (T1) ( $\mathrm{p}=0,0287)$ y al final del último ciclo de soya (T2) $(\mathrm{p}=0,019)$.

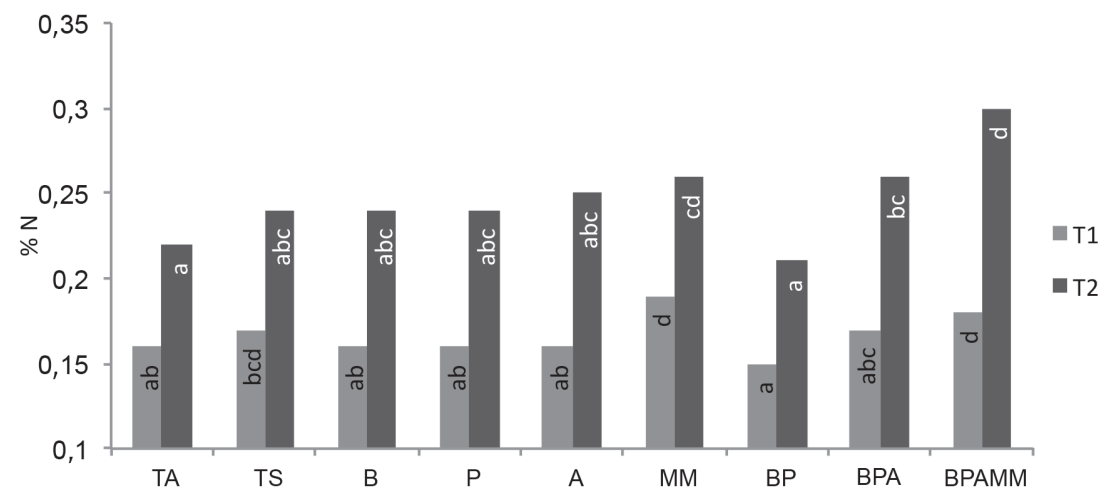

Barras del mismo color con diferentes letras implican diferencias significativas entre los tratamientos.

Fig. 3. Porcentaje de $\mathrm{N}$ del suelo al final del primer ciclo de soya (T1) $(\mathrm{p}=0,0089)$ y al final del último ciclo de soya (T2) $(\mathrm{p}=0,005)$. 
El aumento en el contenido de $\mathrm{C}$ y $\mathrm{N}$ observado en este experimento, se debió a la conversión en biomasa vegetal en el caso del $\mathrm{C}$ y fijación biológica en el caso del $\mathrm{N}$, principalmente a través de la incorporación en todos los tratamientos de la biomasa producida en la unidad experimental. Si se asume que este proceso de captura de $\mathrm{C}$ y $\mathrm{N}$ ocurre en los primeros 5 centímetros del suelo (donde se realizó el muestreo) y se extrapola a una hectárea con una densidad aparente de valor uno, la captura de $\mathrm{C}$ en el tratamiento BPAMM correspondería a $4200 \mathrm{~kg} \cdot \mathrm{ha}^{-1}$ más de $\mathrm{C}(15401 \mathrm{~kg} \mathrm{CO}$, capturado) y $640 \mathrm{~kg} \mathrm{ha}^{-1}$ de $\mathrm{N}$ que en el tratamiento testigo. En este sentido Lal (2004) indica que el límite crítico de carbono orgánico del suelo para la mayoría de los suelos del trópico es de $1,1 \%$ y que hasta los pequeños incrementos se convierten en un gran desafío en los suelos tropicales, aunque Alvarado (2006) demuestra como este valor varía en Costa Rica por zona de vida (entre $1,1-19,8)$ y orden de suelo (entre 3,5-10,9).

Sandoval (2014) encontró que en fincas de café con sombra evaluadas por 28 años en Costa Rica, el incremento en el CO del suelo fue de $0,76 \%$ bajo un manejo orgánico y de $0,60 \%$ para el sistema convencional. Lal (2004) indica que recuperar el carbono orgánico de un suelo puede llevar entre 20-50 años según la textura, la estructura, la temperatura, el sistema del cultivo y el manejo del suelo, mientras que Hungria et ál. (2009) determinó en Brasil que con la adopción de cero labranza se incrementó entre un $25 \%$ y un $30 \%$ el total de $\mathrm{C}$ del suelo a los 7 años y luego de 21 años los incrementos alcanzaron un $45 \%$. Por su parte Ncube et ál. (2011) reporta que aplicaciones combinadas de microorganismos eficientes (ME) en el cultivo del tomate, incrementaron el contenido de $\mathrm{N}$ en la planta y los contenidos de $\mathrm{N}$ en el suelo.

Las variables $\mathrm{pH}$, conductividad eléctrica y respiración de suelos presentaron interacción entre los tratamientos y tiempo trascurrido.

El valor del $\mathrm{pH}$ en el suelo original fue de 5,5 (Cuadro 1), al realizar la evaluación en T1 (Figura 4) se observó una tendencia al incremento del pH, excepto en los tratamientos a los que se aplicó $\mathrm{MM}$, donde el $\mathrm{pH}$ fue significativamente menor, debido posiblemente a la acidez del producto el cual presenta al ser aplicado un $\mathrm{pH}$ alrededor de los 4,0. En la evaluación T2, el pH aumentó significativamente en todos los tratamientos respecto a la evaluación inicial (Figura 4), los tratamientos a los que se aplicó

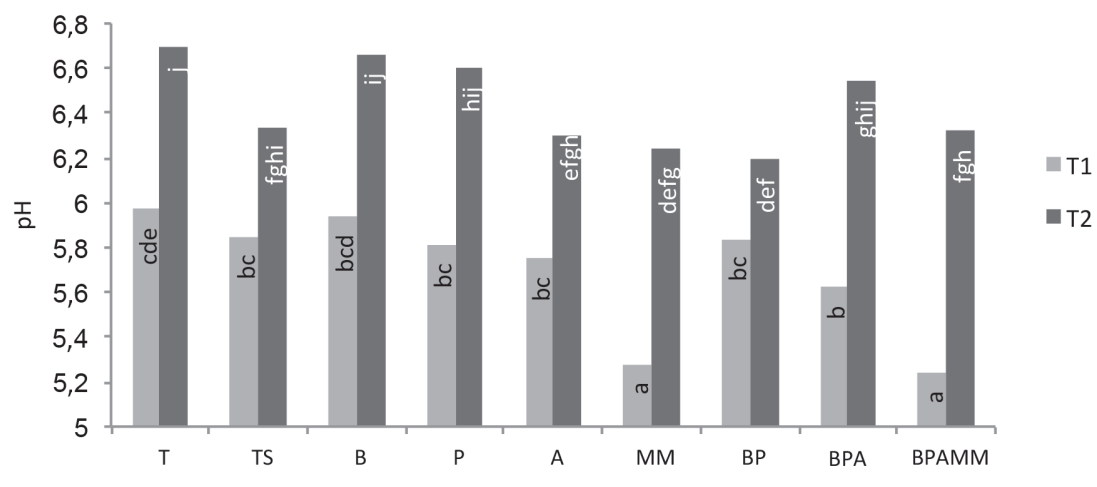

Diferentes letras implican diferencias significativas.

Fig. 4. Valores de $\mathrm{pH}$ al final del primer ciclo de soya (T1) y al final del último ciclo de soya (T2). (Interacción TratamientosTiempo $\mathrm{p}=0,05)$. 
MM muestran un $\mathrm{pH}$ muy similar a los otros tratamientos aunque menores al tratamiento $\mathrm{T}$. El incremento del $\mathrm{pH}$ a lo largo del ensayo se debe posiblemente a la acumulación de elementos solubles, en este caso bases en el sistema cerrado que representa cada unidad experimental. Debido al aumento en las bases, la conductividad eléctrica fue mayor en la mayoría de los tratamientos en T2 (Figura 5), sin que el valor represente un problema para el desarrollo de las plantas, destaca la mayor CE de los tratamientos con adición de MM, posiblemente esto se deba a una mayor solubilización de bases, las cuales se han acumulado en la solución del suelo o al aporte del biol de $\mathrm{MM}$ el cual presentó al ser aplicado una CE de 7,0 dS.m ${ }^{-1}$ (Cuadro 3) debido a los aportes de iones del material orgánico (Bautista et ál. 2000), así como de elementos como Ca y K aplicados en la melaza (Fajardo y Sarmiento 2007). En este sentido Bautista et ál. (2000) reportaron conductividades eléctricas de hasta $19 \mathrm{dS} . \mathrm{m}^{-1}$ en material orgánico líquido tipo vinazas, las mismas se aplicaron al suelo donde no ocasionaron problemas de salinidad. Los autores indican que al interactuar con el suelo se neutralizaron los iones $\mathrm{H}^{+}$y $\mathrm{OH}^{-}$que junto con $\mathrm{K}, \mathrm{Ca}, \mathrm{Mg}$, otros iones y materia orgánica disuelta contribuyen en los valores de la CE.

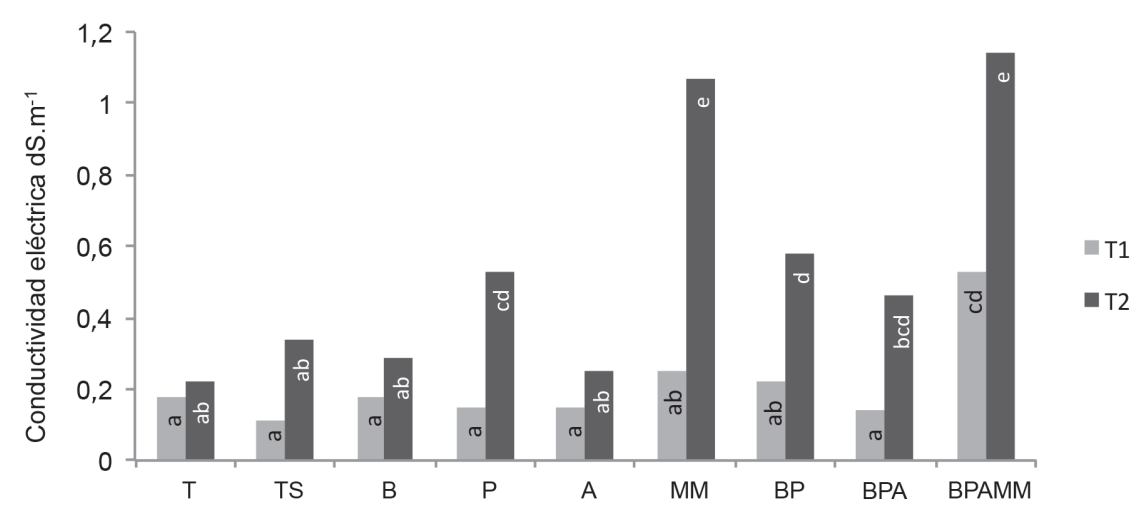

Diferentes letras implican diferencias significativas.

Fig. 5. Valores conductividad eléctrica al final del primer ciclo de soya (T1) y al final del último ciclo de soya (T2). (Interacción Tratamientos-Tiempo p=0,0005).

Cuadro 3. Análisis químico de los bioles de MM listos para la aplicación.

\begin{tabular}{|c|c|c|c|c|c|c|c|c|c|c|c|c|c|c|}
\hline \multirow[b]{2}{*}{ Origen } & \multirow[b]{2}{*}{$\mathrm{pH}$} & \multirow{2}{*}{$\frac{\mathrm{CE}}{\mathrm{dS} . \mathrm{m}^{-1}}$} & \multicolumn{6}{|c|}{$\%$} & \multicolumn{5}{|c|}{$\mathrm{mg} \cdot \mathrm{kg}^{-1}$} & \multirow{2}{*}{$\frac{\text { g.ml }{ }^{-1}}{\text { Dens }}$} \\
\hline & & & $\mathrm{N}$ & $\mathrm{P}$ & $\mathrm{Ca}$ & $\mathrm{Mg}$ & K & S & $\mathrm{Fe}$ & $\mathrm{Cu}$ & $\mathrm{Zn}$ & Mn & B & \\
\hline Río Frío & 3,5 & 7,2 & 0,05 & 0,01 & 0,03 & 0,01 & 0,14 & 0,02 & 39 & ND & ND & ND & ND & 1,01 \\
\hline Coronado & 3,7 & 7,1 & ND & 0,01 & 0,06 & 0,02 & 0,17 & 0,03 & 28 & 1 & 2 & 4 & ND & 1,01 \\
\hline
\end{tabular}


Se observó también un ligero aumento de la CE de los tratamientos con aplicación de $P$. fluorescens que se puede atribuir a la liberación de nutrientes asociadas a las inoculaciones con bacterias promotoras del crecimiento (Bhattacharyya y Jha 2012).

La actividad microbiana (Figura 6) medida como respiración de suelo fue mayor en los tratamientos con presencia de $\mathrm{MM}$ en $\mathrm{T} 1$, mientras que para la evaluación en T2 todos los tratamientos provocaron aumentos en esta variable, al parecer al menos el hecho de mantener el suelo sin disturbar (comparado con el tratamiento T) es suficiente para promover una respuesta, en este caso positiva. Destaca el hecho que los tratamientos con mayor complejidad en orden

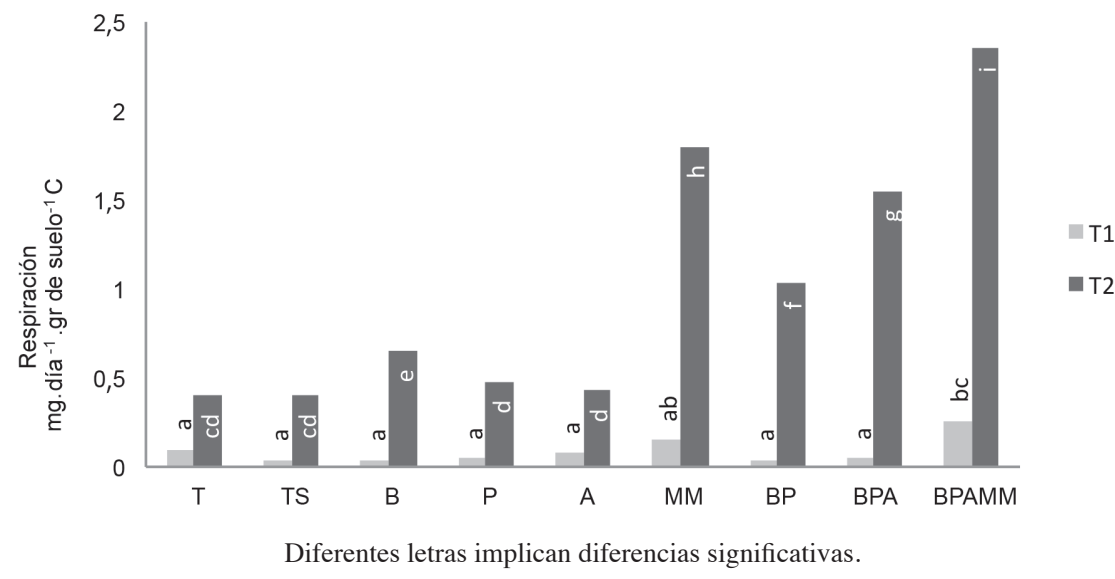

Fig. 6. Respiración de suelo al final del primer ciclo de soya (T1) y al final del último ciclo de soya (T2) (Interacción Tratamientos-Tiempo $\mathrm{p}=<0,0001)$.

ascendente BP, BPA, MM, BPAMM lograron los valores mayores de respiración de suelos en T2. Esto probablemente se deba a que a mayor diversidad microbiana, mayor probabilidad de explotar diferentes fuentes de carbono.

Al evaluar el efecto de los tratamientos sobre las poblaciones de hongos, bacterias y actinomicetes (Cuadro 4) se observó una menor población de microorganismos al comparar el tratamiento $\mathrm{T}$ con los tratamientos TS (presencia de Bradyrhizobium) y A (presencia de Bradyrhizobium y Azospirillum), en este sentido Bashan y de-Bashan (2010) indican que géneros como Azospirillum pueden presentar actividad antagónica debido a la producción de sideróforos, HCN, bacteriocinas y ácido fenilacético. Las

Cuadro 4. Logaritmo del número de UFC/g de hongos, bacterias y actinomicetes evaluados al final del último ciclo de soya $(\mathrm{p}=<0,0001$ para las 3 variables).

\begin{tabular}{|c|c|c|c|c|c|c|c|c|c|}
\hline \multirow{2}{*}{$\begin{array}{l}\text { Logaritmo } \\
\text { del número }\end{array}$} & \multicolumn{9}{|c|}{ Tratamientos } \\
\hline & $\mathrm{T}$ & $\mathrm{TS}$ & B & $P$ & A & MM & $\mathrm{BP}$ & BPA & BPAMM \\
\hline Hongos & $5,60 \mathrm{c}$ & $5,18 \mathrm{ab}$ & $5,44 b c$ & $5,56 \mathrm{c}$ & $5,13 \mathrm{a}$ & $5,95 \mathrm{~d}$ & $5,43 \mathrm{bc}$ & $5,63 \mathrm{c}$ & $5,39 \mathrm{~d}$ \\
\hline Bacterias & $6,65 \mathrm{bc}$ & $6,35 \mathrm{a}$ & $6,68 \mathrm{bcd}$ & $6,87 \mathrm{~cd}$ & $6,33 \mathrm{a}$ & $6,91 \mathrm{~d}$ & $6,61 \mathrm{~b}$ & $6,76 \mathrm{bcd}$ & $7,44 \mathrm{e}$ \\
\hline Actinos & $6,23 \mathrm{~b}$ & $5,95 \mathrm{a}$ & $6,45 \mathrm{bc}$ & $6,67 \mathrm{~d}$ & $5,84 \mathrm{a}$ & $6,74 \mathrm{de}$ & $6,59 \mathrm{~cd}$ & $6,70 \mathrm{cde}$ & $6,95 \mathrm{e}$ \\
\hline
\end{tabular}


inoculaciones con Bacillus y Pseudomonas no provocaron efectos significativos en las poblaciones de hongos, bacterias y actinomicetes, mientras que las inoculaciones mixtas de estos microorganismos lograron aumentar la población de actinomicetes; para las 3 variables mencionadas el tratamiento con mayor complejidad (BPAMM) mostró los recuentos más altos.

Respecto a la producción de biomasa para soya (Figura 7), la inoculación con A, BP y BPA logró aumentar la biomasa evaluada como peso fresco foliar acumulado (suma de la biomasa de todos los ciclos de cultivo) (PFF), en 18\%, 22\% y $23 \%$ respectivamente y en referencia al tratamiento TS, sin embargo, en concordancia con las otras variables evaluadas, fueron los tratamientos con MM los que causaron una mayor producción de biomasa en los 3 ciclos de soya (64\%).

En el caso de tomate (Figura 8) los tratamientos con adición de MM obtuvieron la mayor producción de biomasa (alrededor de 179\%) respecto al tratamiento T. Las inoculaciones con $\mathrm{P}$, BP y BPA también lograron aumentar la producción de biomasa evaluada como PFF acumulado en $43 \%, 43 \%$ y $39 \%$ respectivamente.

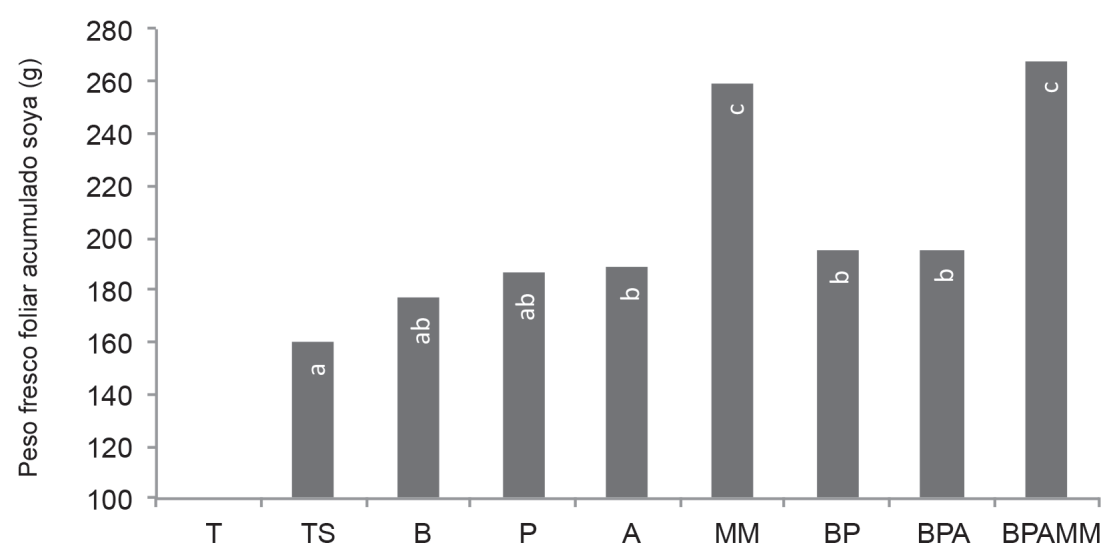

Fig. 7. Peso fresco foliar acumulado obtenido en los 3 ciclos de soya. $(\mathrm{p}=<0,0001)$.

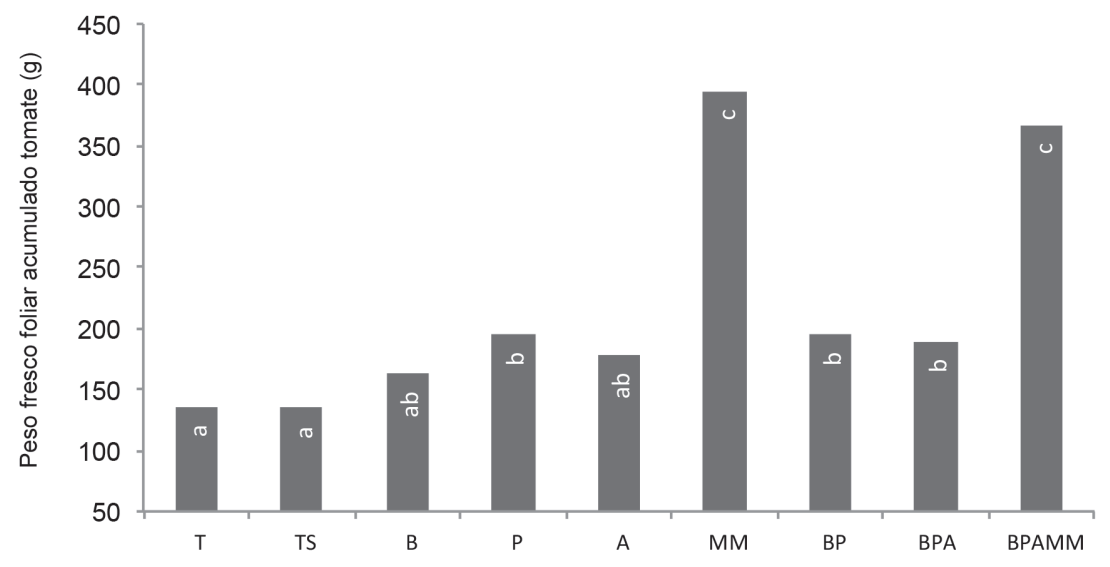

Fig. 8. Peso fresco foliar acumulado obtenido en los 2 ciclos de tomate. $(\mathrm{p}=<0,0001)$. 
Se observó respuesta en el crecimiento de ambos cultivos a las inoculaciones tanto de microorganismos como de bioles de MM, sin embargo la respuesta en porcentajes fue mayor en el caso del tomate; este comportamiento puede deberse a que la soya presenta una relación simbiótica que le permite acceder al nitrógeno atmosférico, elemento considerado como el nutriente más limitante para el crecimiento de las plantas (Franche et ál. 2009), en este sentido Alvarado
(2015) indica que al determinar los contenidos de $\mathrm{N}$ en tejidos foliares de forestales, aquellos que pertenecen a las leguminosas presentan mayores concentraciones.

En cuanto a la cantidad de elementos y microelementos extraídos por las plantas de tomate en el último ciclo, los datos se muestran en el Cuadro 5. Para la mayoría de los nutrientes la inoculación con cualquiera de los microorganismos utilizados logró incrementar el contenido

Cuadro 5. Nutrimentos extraídos por planta en los diferentes tratamientos en el segundo ciclo de tomate (Solanum lycopersicum).

\begin{tabular}{|c|c|c|c|c|c|c|c|c|c|}
\hline \multirow{2}{*}{ g.planta ${ }^{-1}$} & \multicolumn{9}{|c|}{ Tratamientos } \\
\hline & TA & TS & B & $\mathrm{P}$ & A & MM & $\mathrm{BP}$ & BPA & BPAMM \\
\hline $\mathrm{P}$ & $0,01 \mathrm{a}$ & $0,01 \mathrm{a}$ & $0,02 \mathrm{a}$ & $0,02 \mathrm{a}$ & $0,02 \mathrm{a}$ & $0,13 \mathrm{~b}$ & $0,02 \mathrm{a}$ & $0,03 \mathrm{a}$ & $0,13 \mathrm{~b}$ \\
\hline $\mathrm{N}$ & $0,11 \mathrm{a}$ & $0,16 \mathrm{ab}$ & $0,23 \mathrm{bc}$ & $0,28 \mathrm{c}$ & $0,23 \mathrm{bc}$ & $0,53 \mathrm{~d}$ & $0,26 \mathrm{c}$ & $0,28 \mathrm{c}$ & $0,51 \mathrm{~d}$ \\
\hline $\mathrm{Ca}$ & $0,12 \mathrm{a}$ & $0,15 \mathrm{ab}$ & $0,23 \mathrm{bc}$ & $0,26 \mathrm{~cd}$ & $0,24 \mathrm{~cd}$ & $0,64 \mathrm{e}$ & $0,30 \mathrm{~cd}$ & $0,31 \mathrm{~d}$ & $0,65 \mathrm{e}$ \\
\hline $\mathrm{Mg}$ & $0,03 \mathrm{a}$ & $0,06 \mathrm{~b}$ & $0,06 \mathrm{~b}$ & $0,07 \mathrm{~b}$ & $0,06 \mathrm{~b}$ & $0,17 \mathrm{c}$ & $0,08 \mathrm{~b}$ & $0,07 \mathrm{~b}$ & $0,17 \mathrm{c}$ \\
\hline K & $0,22 \mathrm{a}$ & $0,34 \mathrm{~b}$ & $0,33 a b$ & $0,43 \mathrm{~b}$ & $0,35 \mathrm{~b}$ & $0,97 \mathrm{c}$ & $0,45 \mathrm{~b}$ & $0,44 \mathrm{~b}$ & $0,97 \mathrm{c}$ \\
\hline S & $0,06 \mathrm{a}$ & $0,08 \mathrm{a}$ & $0,14 \mathrm{~b}$ & $0,15 \mathrm{~b}$ & $0,14 \mathrm{~b}$ & $0,37 \mathrm{c}$ & $0,17 \mathrm{~b}$ & $0,17 \mathrm{~b}$ & $0,36 \mathrm{c}$ \\
\hline $\mathrm{Fe}$ & $0,98 \mathrm{a}$ & $1,12 \mathrm{a}$ & $2,87 \mathrm{abc}$ & $2,60 \mathrm{abc}$ & $2,26 a b$ & $7,55 \mathrm{~d}$ & $3,29 \mathrm{bc}$ & $4,39 \mathrm{c}$ & $7,06 \mathrm{~d}$ \\
\hline $\mathrm{Cu}(\mathrm{mg})$ & $0,13 \mathrm{a}$ & $0,21 \mathrm{ab}$ & $0,29 \mathrm{bc}$ & $0,35 \mathrm{~cd}$ & $0,30 \mathrm{bc}$ & $0,45 \mathrm{de}$ & $0,35 \mathrm{~cd}$ & 0,37 cde & $0,47 \mathrm{e}$ \\
\hline B (mg) & $0,26 \mathrm{a}$ & $0,41 \mathrm{a}$ & $0,77 \mathrm{~b}$ & $0,84 \mathrm{bc}$ & $0,72 \mathrm{~b}$ & $1,06 \mathrm{c}$ & $0,76 \mathrm{~b}$ & $0,85 \mathrm{bc}$ & $1,07 \mathrm{c}$ \\
\hline
\end{tabular}

$\mathrm{p}=<0,0001$.

foliar del elemento, sin embargo fueron los tratamientos inoculados con MM los que presentaron las respuestas más significativas. Respecto al contenido de $\mathrm{P}$ los únicos tratamientos que no presentaron síntomas visibles de deficiencia de $\mathrm{P}$ (reconocidos como tonalidades púrpuras por el haz y el envés de las hojas bajeras) fueron aquellos a los cuales se les aplicó MM, lo que coincide con los mayores contenidos de $\mathrm{P}$ foliar, posiblemente este hecho está relacionado con las altas poblaciones de microorganismos solubilizadores de $\mathrm{P}$ encontrados en este producto $\left(3,3 * 10^{9} \mathrm{UFC}\right.$. $\mathrm{ml}^{-1}$ ), a la presencia de ácidos orgánicos en el biol que pueden solubilizar el $\mathrm{P}$ presente (Richardson et ál. 2009), o a un efecto combinado de ambos factores. Cuando se comparó el contenido de P en el suelo original, con el contenido de $\mathrm{P}$ en el tratamiento con MM al final del ensayo (Cuadro 6), se encontró que a pesar de los ciclos de cultivo, este elemento pasó de 6 a $20 \mathrm{mg} . \mathrm{l}^{-1}$ mientras que en el tratamiento $\mathrm{T}$ al final del ensayo fue de $3 \mathrm{mg} . \mathrm{l}^{-1}$. 
Cuadro 6. Análisis químico del suelo original, de los tratamientos BPAMM y T, al final del ensayo.

\begin{tabular}{|c|c|c|c|c|c|c|c|c|c|c|c|c|}
\hline & \multirow{2}{*}{$\begin{array}{c}\mathrm{pH} \\
\mathrm{H}_{2} \mathrm{O}\end{array}$} & \multicolumn{5}{|c|}{$\operatorname{cmol}(+) \cdot 1^{-1}$} & \multirow{2}{*}{$\begin{array}{c}\% \\
\text { SA }\end{array}$} & \multicolumn{5}{|c|}{$\mathrm{mg} . \mathrm{l}^{-1}$} \\
\hline & & ACIDEZ & $\mathrm{Ca}$ & $\mathrm{Mg}$ & $\mathrm{K}$ & CICE & & $\mathrm{P}$ & $\mathrm{Zn}$ & $\mathrm{Cu}$ & $\mathrm{Fe}$ & $\mathrm{Mn}$ \\
\hline Suelo original & 5,5 & 0,1 & 7,2 & 1,4 & 0,5 & 9,3 & 2,0 & 6,0 & 8,0 & 3,0 & 275 & 115 \\
\hline BPAMM * & 6,2 & 0,2 & 6,1 & 2,0 & 1,2 & 9,4 & 1,8 & 19,8 & 4,8 & 3,3 & 189 & 18 \\
\hline $\mathrm{T}^{*}$ & 5,9 & 0,2 & 5,5 & 1,4 & 0,3 & 7,4 & 2,3 & 3,3 & 4,2 & 2,0 & 171 & 34 \\
\hline
\end{tabular}

*Promedio de 4 repeticiones.

En el caso del N la inoculación con B, BP y BPA (comparado con TS) causó un aumento del contenido foliar de este elemento, pero fue la aplicación de MM el tratamiento que permitió obtener los mayores contenidos de $\mathrm{N}$ en la planta (Cuadro 5).

Los tratamientos a los que se aplicó MM presentaron los mayores valores de $\mathrm{Mg}, \mathrm{K}, \mathrm{Ca} \mathrm{y}$ $\mathrm{S}$ (Cuadro 5), mientras que la rotación con soya logró aumentar la cantidad de Mg y K absorbida por la planta, no así las inoculaciones con microorganismos solos o en mezcla. Para el Ca la inoculación con P, A, BP y BPA aumentó el nivel de este elemento en la planta; en el caso del S la inoculación con al menos un microorganismo aumentó la absorción del nutriente. En el Cuadro 6 se observa el aumento en los contenidos de $\mathrm{Mg}$ y $K$ en la solución del suelo en el tratamiento BPAMM al final del ensayo, mientras que el $\mathrm{Zn}$, Fe y el Mn generalmente relacionados con condiciones reducidas y ácidas en el suelo, disminuyeron tanto en el tratamiento BPAMM como en el tratamiento $\mathrm{T}$.

Para los microelementos $\mathrm{Fe}, \mathrm{Cu}, \mathrm{B}$ los tratamientos con aplicación de MM fueron también los que permitieron un mayor contenido del elemento en la planta (Cuadro 5). En el caso de las inoculaciones con los microorganismos, para el Fe solamente los tratamientos BP y BPA aumentaron los valores de este elemento con respecto al tratamiento $\mathrm{T}$, mientras que las inoculaciones con un solo microorganismo no presentaron respuesta; para el $\mathrm{Cu}$ los tratamientos $\mathrm{P}, \mathrm{A}$, BP y BPA lograron incrementar la absorción del elemento respecto a T y a TS; para el caso del B la inoculación con al menos un microorganismo aumentó la absorción del nutriente.

Richardson et ál. (2009) indica que la inoculación con rizobacterias aumenta la absorción de nutrientes como $\mathrm{Ca}, \mathrm{K}, \mathrm{Fe}, \mathrm{Cu}, \mathrm{Mn}$ y $\mathrm{Zn}$ y que este incremento podría ser explicado por la producción de ácidos orgánicos por las plantas y/o las bacterias que disminuye el pH de la rizósfera y hacen solubles formas no disponibles de nutrimentos adyacentes a la raíz. En el caso del $\mathrm{P}$ pueden alterar la solubilidad de compuestos inorgánicos o afectar la cinética de las reacciones de adsorción-desorción de ortofosfatos en el suelo con la subsecuente disponibilidad de fosfatos. Otros factores a considerar son el potencial de oxidación/reducción y el pH del suelo.

Hay que destacar que la aplicación de MM afectó una cantidad importante de las variables evaluadas, las cuales convergen en una mayor producción de biomasa de soya y tomate. Este tratamiento modificó las propiedades biológicas del suelo según quedó evidenciado al evaluar la respiración y las poblaciones microbianas, este efecto puede deberse al aporte nutricional del MM a la flora microbiana del suelo consistente en nutrientes (Cuadro 6), azúcares, vitaminas, ácidos orgánicos, y a la adición de un inóculo microbiano rico en levaduras $\left(1 * 10^{8}\right.$ UFC. $\left.\mathrm{ml}^{-1}\right)$ y lactobacilos $\left(1,5^{*} 10^{8}\right.$ UFC.ml $\left.{ }^{-1}\right)$, microorganismos que producen ácidos orgánicos, antibióticos y sustancias promotoras de crecimiento. El aporte nutricional directo a la planta es poco probable 
ya que el producto al ser aplicado presenta muy bajos contenidos de nutrimentos (Cuadro 3).

Por otro lado, la aplicación de MM favoreció una mayor absorción de nutrientes por las plantas y/o una mayor disponibilidad de $\mathrm{P}, \mathrm{Mg}$ y K en la solución del suelo (Cuadro 6). Estos efectos pueden deberse a la liberación de ácidos orgánicos durante la descomposición de residuos, al mayor aporte de biomasa en este tratamiento, a cambios en el pH del suelo y a cambios en la flora microbiana que incidieran en la promoción de crecimiento. Según Nakkeeran (2005), la aplicación de mezclas de cepas compatibles de bacterias promotoras de crecimiento podría imitar el entorno natural si se amplía el espectro de acción de las inoculaciones microbianas que permitan así efectos aditivos que pueden incrementar la producción y el crecimiento vegetal. Bashan et ál. (2004) indican que las co-inoculaciones permiten a la planta una nutrición mas balanceada, y/o una mayor absorción de nutrientes.

Según los resultados de este estudio la utilización de bioles de MM afectó las propiedades químicas y biológicas de las unidades experimentales y este efecto promovió el crecimiento de las plantas de soya y tomate. Por su origen (poblaciones nativas de bosques naturales) y preparación, los bioles de MM tienen potencial para ser utilizados como insumo en la producción agrícola sostenible. Es necesaria una mayor investigación de este inoculo microbiano que nos permita identificar a nivel molecular los consorcios microbianos presentes en las diferentes etapas del proceso de elaboración y los mecanismos por los cuales afectan positivamente el crecimiento de las plantas.

\section{AGRADECIMIENTOS}

A los Laboratorios de Suelos y Foliares, Recursos Naturales y Microbiología Agrícola del Centro de Investigaciones Agronómicas de la Universidad de Costa Rica por su financiamiento para realizar los análisis. A la Vicerrectoría de Investigación por financiar el proyecto VI733-B1-129 que generó el presente artículo.

\section{LITERATURA CITADA}

ALVARADO A. 2006. Potential of soil carbon sequestration in Costa Rica. Chapter 8, pp. 147-165. In: R. La.1, C.C. Cerri, M. Bernoux, J. Etchevers and C.E. Cerri (eds.). Carbon sequestration in soils of Latin America. The Haworth Press Inc. USA.

ALVARADO A., CAMACHO M., FERNÁNDEZ J., MEZGER G., MATA R., BERTSCH F., ARAYA M., AVELLÁN M., MURILLO R., RAMÍREZ D., PORTUGuez E., FAllas J., ÁVIlA C., MONTERO M., RAIGOSA J., RÍOS V., VAIDES E. 2015. Interpretación del análisis foliar de varias especies forestales latifoliadas del trópico americano. 61 p. In: VIII Congreso Nacional de Suelos. Asociación Costarricense de la Ciencia del Suelo. San José, Costa Rica.

BALOTA E., COLOZZI A., ANDRADE D., DICK R. 2004. Long-term tillage and crop rotation effects on microbial biomassand $\mathrm{C}$ and $\mathrm{N}$ mineralization in a Brazilian Oxisol. Soil \& Tillage Research 77:137145.

BARRIOS E. 2007. Soil Biota, Ecosystem Services and Land Productivity. Ecological Economics 64:269-285.

BASHAN Y., DE-BASHAN L. 2010. Chapter 2. How the plant growth-promoting bacteria Azospirillum promotes plant growth-A critical assessment. Advances in Agronomy 108:77-136.

BASHAN Y., HOLGUIN G., DE-BASHAN L. 2004. Azospirillum-plant relationships: physiological, molecular, agricultural, and environmental advances (1997-2003). Can. J. Microbiol. 50:521-577.

BAUTISTA F., DURÁN C., LOZANO R. 2000. Cambios químicos en el suelo por aplicación de materia orgánica soluble tipo vinazas. Rev. Int. Contam. Ambient. 16(3):89-101.

BAUTISTA J., GARCÍA R., PÉREZ J., ZAVALETA E., MONTES R., FERRERA R. 2008. Inducción de supresividad a fitopatógenos del suelo. Un enfoque holístico al control biológico. ITERCIENCIA 33(2):96-102.

BHATTACHARYYA P., JHA K. 2012. Plant growthpromoting rhizobacteria (PGPR): emergence in agriculture. World J Microbiol Biotechnol. 28:13271350.

BREMNER J., TABATABAI M. 1971. Use of automated combustion techniques for total carbon, total nitrogen, and sulfur analysis of soils, pp. 1-16. In: L.M. Walsh (ed.). Instrumental methods for analysis of soils and plant tissue.

CAPRA F. 1998. La trama de la vida. Una nueva perspectiva de los sistemas. Trad. Sempau D. 2 Ed. Anagrama. Barcelona, España. 367 p.

CASTRO R., CORNEJO H., RODRÍGUEZ L., BUCIO J. 2009. The role of microbial signals in plant growth and development. Plant Signal Behav. 4(8):701-712. 
CHAPARRO J., SHEFLIN A., MANTER D., VIVANCO J. 2012. Manipulating the soil microbiome to increase soil health and plant fertility. Biol Fertil Soils 48:489-499.

CUMMINGS S. 2009. The application of plant growth promoting rhizobacteria (PGPR) in low input and organic cultivation ofgraminaceous crops; potential and problems. Environ Biotechnol 5:43-50.

DE VLEESSCHAUWER D., HÖFTE M. 2009. Rhizobacteria-induced systemic resistance, pp. 223281. In: L.C. Van Loon (ed.). Advances in botanical research, vol 51. Elsevier, Burlington.

DÍAZ R., HUNTER A. 1978. Metodologías de muestreo de suelos, análisis químico de suelos y tejido vegetal y de investigación en invernadero. CATIE. Turrialba, Costa Rica. 2 p.

DIMKPA C., WEINAND T., ASCH F. 2009. Plantrhizobacteria interactions alleviate abiotic stress condition. Plant, Cell and Environment 32:16821694.

EHRENFELD J., RAVIT B., ELGERSMA K. 2005. Feedback In The Plant-Soil System. Annu. Rev. Environ. Resour. 30:75-115.

EISENHAUER N., BEBLER H., ENGELS C., GLEIXNER G., HABEKOST M., MILCU A., PARTSCH S., SABAIS A., SCHERBER C., STEINBEISS S., WEIGELT A., WEISSER W., SCHEU S. 2010. Plant diversity effects on soil microorganisms support the singular hypothesis. Ecology 91(2):485-496.

FAJARDO E., SARMIENTO S. 2007. Evaluación de la melaza de caña como sustrato para la producción de Saccharomyces cerevisiae. Tesis de licenciatura, Universidad Javeriana, Colombia. 120 p.

FORSYTHE W. 1975. Física de suelos. Manual de Laboratorio. IICA, Costa Rica. 212 p.

FRANCHE C., LINDSTRÖM K., ELMERI C. 2009. Nitrogen-fixing bacteria associated with leguminous and non-leguminous plants. Plant Soil 321:35-59.

GODFRAY C., BEDDINGTON J., CRUTE I., HADDAD L., LAWRENCE D., MUIR J., PRETTY P., ROBINSON S., THOMAS S., TOULMIN C. 2010. Food Security: The Challenge of Feeding 9 Billion People. Science 327:812-818.

GOVAERTS B., MEZZALAMA M., UNNO Y., SAYRE K., LUNA M., VANHERCK K., DENDOOVEN L., DECKERS J. 2007. Influence of tillage, residue management, and crop rotation on soil microbial biomass and catabolic diversity, Appl. Soil Ecol 37:18-30.

GUIÑAZÚ L., ANDRÉS J., DEL PAPA M., PISTORIO M., ROSAS S. 2009. Response of alfalfa (Medicago sativa L.) to single and mixed inoculation with phosphate-solubilizing bacteria and Sinorhizobium meliloti. Biol Fertil Soils 46:185-190.
HARTEMINK A.E. 2003. Soil fertility decline in the tropics with case studies on plantations. CABI Publishing, England. $360 \mathrm{p}$.

HENRÍQUEZ C., CABALCETA G. 2012. Guía práctica para el estudio introductorio de los suelos con un enfoque agrícola. Asociación Costarricense de la Ciencia del Suelo. San José, Costa Rica. 111 p.

HUNGRIA M.,FRANCHINIJ.,BRANDA OO., KASCHUK G., ROSINEI R. 2009. Soil microbial activity and crop sustainability in a long-term experiment with three soil-tillage and two crop-rotation systems. Applied Soil Ecology 42:288-296.

JENKINSON D., POWLSON D. 1976. The effects of biocidal treatment on metabolism in soil-V. A method for measuring soil biomass. Soil Biology and Biochemistry 8(3):209-213.

JONES D., HINSINGER P. 2008. The rhizosphere: complex by design. Plant Soil 312:1-6.

KEMPER W.D. 1965. Aggregate stability, pp. 511-519. In: C.A. Black, D.D. Evans, J.L. White, L.E. Ensminger and F.E. Clark (eds.). Methods of soil analysis. Part 1. Physical and Mineralogical properties, including statistics of measurement and sampling. American society of Agronomy Inc., publisher. Madison Wisconsin. USA.

KIBBLEWHITE M., RITZ K., SWIFT M. 2015. Soil health in agricultural systems. Philosophical Transactions of the Royal Society B: Biological Sciences 363(1492):685-701.

KIRANKUMAR R., JAGADEESH K., KRISHNARAJ P., PATIL M. 2008. Enhanced growth promotion of tomato and nutrient uptake by plant growth promoting rhizobacterial isolates in presence of tobacco mosaic virus pathogen. Karnataka J. Agric Sci 21:309-311.

LAL R. 2004. Soil carbon sequestration impacts on global climate change and food security. Science 304:16231627.

MARÍN S. 2015. Comparación del comportamiento de un Andisol manejado orgánicamente y otro convencional mediante la respuesta de la papa en invernadero a prácticas orgánicas, convencionales y de solubilizadores de P. VIII Congreso Nacional de Suelos. Asociación Costarricense de la Ciencia del Suelo. San José, Costa Rica, p. 39.

MARTÍNEZ E., FUENTES J., ACEVEDO E. 2008. Carbono Orgánico y Propiedades Del Suelo J. Soil Sc. Plant Nutr. 8(1):68-96.

MAYERA J., SCHEIDA S., WIDMERA F., FLIEßBACHB A., OBERHOLZERA H. 2010. How effective are 'Effective microorganisms ${ }^{\circledR}$ (EM)'? Results from a field study in temperate climate Applied Soil Ecology 46:230-239.

MENDES R., KRUIJT M., DE BRUIJN I., DEKKERS E., VAN DER VOORT M., SCHNEIDER J., PICENO Y., DESANTIS T., ANDERSEN G., BAKKER 
P., RAAIJMAKERS J. 2011. Deciphering the rhizosphere microbiome for disease-suppressive bacteria. Science 332:1097-1100.

MITTER B., BRADER G., AFZAL M., COMPANT S., NAVEED M., TROGNITZ F., SESSITSCH A. 2013. Advances in Elucidating Beneficial Interactions Between Plants, Soil, and Bacteria Birgit Advances in Agronomy 121(7):382-426.

MONTAGNINI F., RAMSTAD K., SANCHO F. 1993. Litterfall, litter decomposition and the use of mulch of four indigenous tree species in the Atlantic lowlands of Costa Rica. Agroforestry Systems 23(1):39-61.

NAKKEERAN S., FERNANDO W., SIDDIQUI Z. 2005. Plant Growth Promoting Rhizobacteria Formulations And Its Scope In Commercialization For TheManagement Of Pests And Diseases, pp. 257-296. In: Siddiqui (ed.), PGPR: Biocontrol and Biofertilization. Chapter 10 CSpringer, Dordrecht, The Netherlands.

NCUBE L., MNKENI P., BRUTSCH M. 2011. Agronomic suitability of effective micro-organisms for tomato production. African Journal of Agricultural Research 6(3):650-654.

RESTREPO J., HENSEL J. 2007. El ABC de la agricultura orgánica, fosfitos y panes de piedra. Jairo Restrepo Riverd. Colombia. 396 p.

RICHARDSON A., BAREA J., McNEILL A., PRIGENT C. 2009. Acquisition of phosphorus and nitrogen in the rhizosphere and plant growth promotion by microorganisms Plant Soil 321:305-339.

SANDOVAL D. 2014. Evaluación De Modelos Estadísticos Para La Estimación Del Carbono Orgánico Del Suelo,
En Sistemas De Producción De Café Orgánicos y Convencionales De La Zona De Caraigres, Costa Rica. Tesis de licenciatura, Universidad de Costa Rica, Turrialba, Costa Rica. 76 p.

SAUERBECK D., GONZÁLEZM. 1977. Field decomposition of carbon-14-labelled plant residues in various soils of the Federal Republic of Germany and Costa Rica, pp. 159-170. In: IAEA and FAO (eds.) in cooperation with Agrochimica Vol. 1. Proc. Symp. Braunschweig.

SINGH J., PANDEY V. 2011. Efficient soil microorganisms: A new dimension for sustainable agriculture and environmental development. Singh Agriculture, Ecosystems and Environment 140:339-353.

TORSVIK V., ØVREÅS L. 2002. Microbial diversity and function in soil: from genes to ecosystems. Ecology and industrial microbiology 5:240-245.

VANCE E., BROOKES P., JENKINSON D. 1987. An extraction method for measuring soil microbial biomass C. Soil Biology and Biochemistry 19(6):703707.

VAN der HEIJDEN M., BARDGETT M., STRAALEN R. 2008. The unseen majority: soil microbes as drivers of plant diversity and productivity in terrestrial ecosystems. Ecol. Lett. 11:296-310.

VESSEY J. 2003. Plant growth promoting rhizobacteria as biofertilizers. Plant Soil (255):571-586.

WOOD T., LAWRENCE D. 2008. No short-term change in soil properties following four-fold litter addition in a Costa Rican rain forest. Plant Soil 307:113-122.

YOUNG I., CRAWFORD J. 2004. Interactions and SelfOrganization in the Soil-Microbe Complex. Science 304:1634-1637.

Todos los derechos reservados. Universidad de Costa Rica. Este artículo se encuentra licenciado con Creative Commons Reconocimiento-NoComercial-SinObraDerivada 3.0 Costa Rica. Para mayor información escribir a rac.cia@ucr.ac.cr 